\title{
Automated 3D renal segmentation based on image partitioning
}

\author{
Varduhi Yeghiazaryan ${ }^{\mathrm{a}}$ and Irina D. Voiculescu ${ }^{\mathrm{a}}$ \\ ${ }^{a}$ Department of Computer Science, University of Oxford, Wolfson Building, Parks Road, \\ Oxford, UK
}

\begin{abstract}
Despite several decades of research into segmentation techniques, automated medical image segmentation is barely usable in a clinical context, and still at vast user time expense. This paper illustrates unsupervised organ segmentation through the use of a novel automated labelling approximation algorithm followed by a hypersurface front propagation method. The approximation stage relies on a pre-computed image partition forest obtained directly from CT scan data. We have implemented all procedures to operate directly on 3D volumes, rather than slice-by-slice, because our algorithms are dimensionality-independent. The results picture segmentations which identify kidneys, but can easily be extrapolated to other body parts. Quantitative analysis of our automated segmentation compared against hand-segmented gold standards indicates an average Dice similarity coefficient of $90 \%$. Results were obtained over volumes of CT data with 9 kidneys, computing both volume-based similarity measures (such as the Dice and Jaccard coefficients, true positive volume fraction) and size-based measures (such as the relative volume difference). The analysis considered both healthy and diseased kidneys, although extreme pathological cases were excluded from the overall count. Such cases are difficult to segment both manually and automatically due to the large amplitude of Hounsfield unit distribution in the scan, and the wide spread of the tumorous tissue inside the abdomen. In the case of kidneys that have maintained their shape, the similarity range lies around the values obtained for inter-operator variability. Whilst the procedure is fully automated, our tools also provide a light level of manual editing.
\end{abstract}

Keywords: image segmentation, CT, hierarchical partitioning, organ labelling, Fast Marching

\section{INTRODUCTION}

In a clinical or clinical research context, the shapes of anatomical structures of interest are typically found from single slices of CT or MRI imaging data using commercial software (such as Mimics ${ }^{1}$ or Simpleware ${ }^{2}$ ). These shapes are segmented from individual images manually: this involves setting a threshold then going through slice by slice and tidying up regions which have not been detected correctly. Processing a set of scan slices takes many user hours. The uptake of computer-based segmentation in research fields which process large volumes of visual data depends crucially on shortening this user time.

In this paper we refer to segmentation as the process of identifying and labelling a 3D region of semantic importance (such as a kidney). In doing so, we pre-process the data in order to group voxels with similar characteristics into regions - a process known in Computer Vision as (2D) image segmentation. In order to avoid ambiguity, we will refer to the vision segmentation technique as partitioning the image, and to the medical segmentation technique as labelling the features of interest.

Hypersurface front propagation methods, like level set and Fast Marching methods, have been previously used for medical image analysis. Malladi et al. ${ }^{3-7}$ use level set methods to identify features in 2D and 3D images. Fast Marching for feature labelling in medical images was investigated in a number of papers. ${ }^{8-12}$

Current practice for medical imaging ${ }^{5,8-10,13}$ leaves the initialisation of level sets or Fast Marching to the user. Even commercial software for processing scan data expects the user to hand-seed any similar methods, and

Further author information: (Send correspondence to I.V.)

I.V.: E-mail: irina@cs.ox.ac.uk

V.Y.: E-mail: varduhi@cs.ox.ac.uk 
this on a slice-by-slice basis. In other cases, heavily data-specific methods are used for automated seed (initial region) choice. ${ }^{11,12}$ We present a new approach for fully automatic labelling and reconstruction of features, such as organs, which combines Fast Marching with a hierarchical image partitioning method.

Following our previous work on decision-support systems for renal segmentation, ${ }^{14,15}$ we illustrate novel unsupervised organ segmentation through the use of an automated initial labelling approximation algorithm followed by a hypersurface front propagation method. The initial approximation stage relies on a pre-computed image partition forest obtained directly from CT scan data.

\section{IMAGE PARTITIONING}

We illustrate our algorithms with abdominal CT scans in DICOM format, each volume typically comprising up to 50 axial slices, $512 \times 512$ voxels each. Our labelling approach is easily generalised to any dimensionality. We choose always to run it on $3 \mathrm{D}$ data volumes, preserving the original resolution. We pre-process the raw data with windowing, ${ }^{16}$ which maps the original radiodensity-based range of Hounsfield Units (HU) to (256) greyscale values preserving granularity detail in a given HU subrange of interest. After this, edge-preserving smoothing with several iterations of anisotropic diffusion filtering ${ }^{17}$ is applied. The pre-processing is followed by a partitioning step, where the 3D data volume is organised into a tree-based representation of adjacency graphs. The nodes of the trees are 3D regions of voxels, and the parental relationship is that of inclusion. Essentially, the data is partitioned into a hierarchy of inter-connected regions and subregions. We call this data structure an image partition forest (IPF). We have described elsewhere ${ }^{18,19}$ how we use a watershed and waterfall transform-based approach ${ }^{20}$ to construct the IPF in a bottom-up manner.

The most crucial aspect of automating the segmentation is the way in which we use the IPF to find a rough approximation of a kidney and seed from it a hypersurface front propagation method which helps localise the kidney boundaries more accurately.

Since the typical HU range (and hence windowed greyscale) for kidneys is known from radiology studies, ${ }^{16}$ we can choose as kidney candidates those regions in the IPF which are within the desired mean greyscale range. Furthermore, filtering regions which correspond roughly to kidneys can also be based on anatomical knowledge. The IPF candidate regions should be in a high layer of the IPF (layers 3-5); in an axial slice, the right kidney should lie 'west' of the spine and anatomically close to it; also, the right kidney should span a reasonable number of voxels (depending on the number of slices in the image). A similar filtering procedure, employing symmetry, has been devised for left kidneys. Other abdominal major organs can also be approximated in the IPF with analogous approaches relying only on established anatomical knowledge.

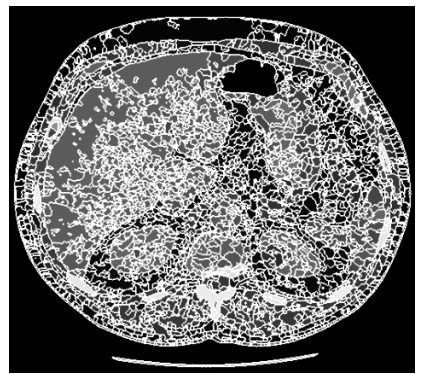

(a)

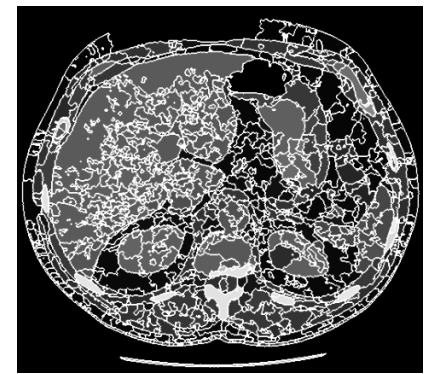

(b)

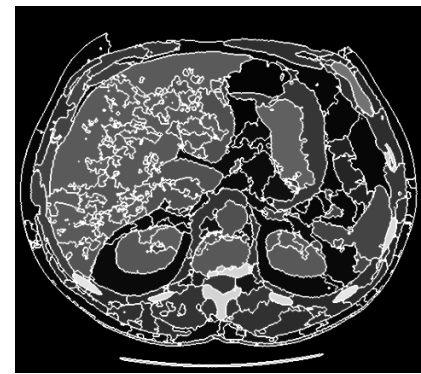

(c)

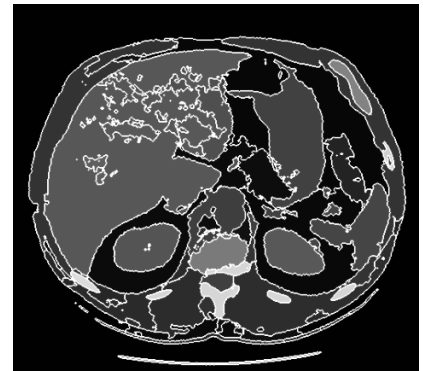

(d)

Figure 1. Four of the six layers (finest layers not shown) in a 3D IPF, representing image partitions in decreasing detail level.

Figure 1 illustrates a 2D projection through a 3D IPF, and its different levels of coarseness. The bottom-most level is made up of the raw image voxels; it is followed by a layer of over-segmented regions (not shown), followed by increasingly coarse regions that belong together and gradually group themselves around organs and other features. 


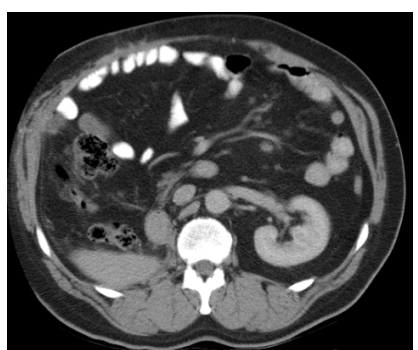

(a) Single abdominal CT slice. Note that the right kidney has undergone nephrectomy.

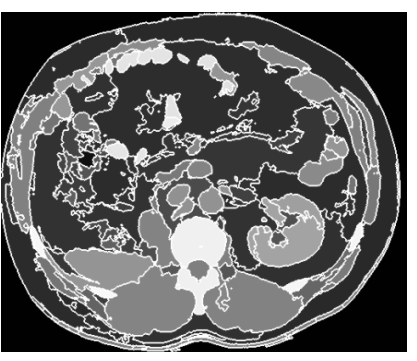

(b) Layer of IPF resulting from the segmentation of (a). Projection on a single slice of the $3 \mathrm{D}$ structure IPF.

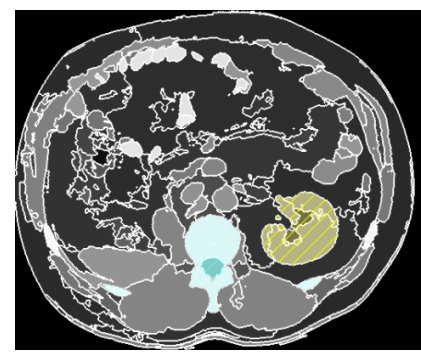

(c) Approximation of the left kidney in the IPF (in yellow). The algorithm did not find a right kidney candidate.

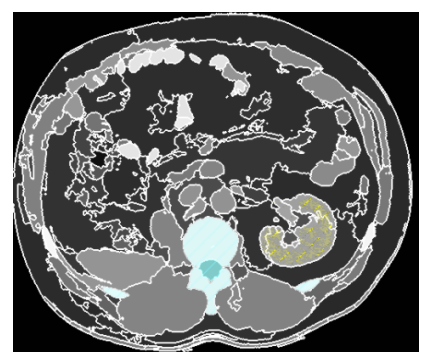

(d) Seed points extracted from regions approximated in (c); only points in healthytissue range for kidney.

Figure 2. Image partitioning and seed extraction from the resulting IPF

\section{FEATURE LABELLING WITH FAST MARCHING AND MORPHOLOGICAL CLOSING}

Our hypersurface front propagation method of choice is Fast Marching - an efficient iterative algorithm, introduced by Sethian, ${ }^{21,22}$ for numerical approximation of the development of fronts propagating through $\mathbb{R}^{n}$ space $\left(\mathbb{R}^{3}\right.$ in this case). An $O(N)$ complexity implementation of Fast Marching can be found in the literature. ${ }^{23,24}$ In the image segmentation literature ${ }^{10}$ this is always seeded manually. Multi-stage approaches to Fast Marching seeded from previous results have been used $^{12}$ but these are very data specific.

The novelty of our approach is that we seed the Fast Marching automatically from a subset of the candidate regions output via the IPF. Our take to the seeding process relies on the automatic generation of the IPF structure. Whilst filtering the candidate regions relies on anatomical structure, this can be hard-coded into the automated segmentation. Only voxels which are in the expected healthy-tissue greyscale range are considered and used as seeds, as shown in Fig. 2(d).

The candidate regions chosen using the IPF do not approximate the kidneys sufficiently well themselves. Local heterogeneities of high gradient magnitude value are usually missed and most regions have displaced edges. Because of this, these candidate regions may miss necessary pieces of tissue or contain chunks of external tissue. This is why the Fast Marching is employed. The quality of the outcome is highly dependent on the speed term and the rate of development of the front. We conducted a range of systematic experiments ${ }^{25}$ with several speed terms previously used in literature for medical image analysis. Our best results were achieved with $F(\vec{x})=e^{-|\nabla I(\vec{x})|}$. Here $|\nabla \cdot|$ is the magnitude of the gradient and $I(\vec{x})$ is the value of the image at location $\vec{x}$ after pre-processing (windowing and smoothing in this case).

Without going into detail, it is easy to see from this speed function that the development of the front slows down at points with high gradient magnitude values (in particular, in the neighbourhood of organ boundaries). We captured such slow development of the front by stopping the growth after the development is less than a threshold percentage of the region's volume in one time step.

The regions output by the Fast Marching are then finalised with several iterations of morphological closing ${ }^{26}$ in order to remove spurious holes and to smooth out the boundaries.

\section{AUTOMATED SEGMENTATION}

Putting all the above elements together, we construct the following novel automated segmentation procedure:

1. Pre-process the data with windowing and anisotropic diffusion filtering (as per $\S 2$ )

2. Partition the data and construct the IPF $(\S 2)$

3. Filter the resulting IPF for candidate kidney regions based on anatomical knowledge ( $(2)$ 
4. Within candidate regions, choose seed points which are in an expected healthy-kidney greyscale range (§3)

5. Choose hypersurface front propagation and its parameters, and seed it from Step $4(\S 3)$

6. Advance the front until it makes little or no progress $(\S 3)$

7. Finalise the labelled regions with several iterations of morphological closing (§3).

Steps 2-4 of the automated method are illustrated on a slice in Fig. 2, and some final results on another slice in Fig. 5(a).

\section{RESULTS AND VALIDATION}

We have carried out extensive experiments on 3D volumes of up to $50 \mathrm{CT}$ slices, allowing for both partitioning and Fast Marching to extend in all three dimensions. We varied the algorithm parameters systematically, and the overall results were consistent with what is summarised here.

Our quantitative evaluation is based on 9 different kidneys (denoted A-I) from 7 patients' CT volumes of 5-38 slices each. Most of these volumes come from nephrectomised patients and contain only one kidney. A knowledgeable operator traced contours manually for each organ. Inter-operator variability of 93-94\% (Dice coefficient) was estimated by asking two different operators to label soft-tissue organs in a single abdominal volume.

Table 1 shows the comparison between the manually traced gold standard and the output of our unsupervised segmentation algorithm, quantified using Dice similarity coefficient (DSC), ${ }^{27}$ Jaccard similarity coefficient (JSC), true positive volume fraction (TPVF), true negative volume fraction (TNVF), precision (Prec) and relative volume difference $(R V D) .{ }^{28-30}$ The Dice coefficient evaluation is split per kidney in Tab. 2 and is close to the inter-observer variability. The evaluation outcome can readily be compared to kidney segmentation results previously reported in the literature. ${ }^{31,32}$

Table 1. Match of our segmentation Table 2. Dice similarity coefficient against gold standards with different simi- of our segmentation and gold stanlarity measures dards per kidney

\begin{tabular}{|l|rrr|}
\hline Sim Measure & Mean & & St dev \\
\hline \hline DSC & 90.37 & \pm & 1.69 \\
\hline \hline JSC & 82.47 & \pm & 2.8 \\
\hline 1-RVD & 92.57 & \pm & 5.98 \\
\hline TPVF & 92.81 & \pm & 4.8 \\
\hline TNVF & 99.86 & \pm & 0.07 \\
\hline Prec & 88.29 & \pm & 2.47 \\
\hline
\end{tabular}

\begin{tabular}{|l|r|}
\hline Kidney & DSC \\
\hline A & 93.016 \\
B & 91.528 \\
C & 91.394 \\
D & 91.025 \\
E & 90.824 \\
F & 90.360 \\
G & 90.024 \\
H & 87.752 \\
I & 87.396 \\
\hline
\end{tabular}

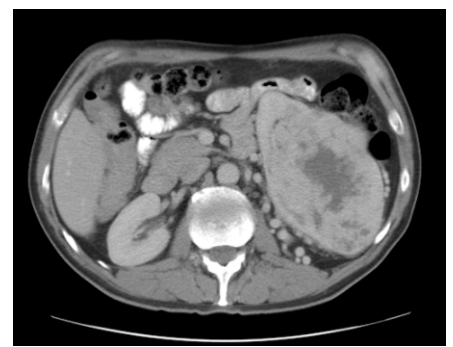

Figure 3. An extreme case of a tumourous left kidney on which our algorithm yields only $30 \%$ Dice similarity

Our dataset contains images of low resolution (5mm slice thickness, $0.68-0.78 \mathrm{~mm}$ pixel resolution), acquired with or without contrast agent administration and covers both healthy and diseased kidneys. However, we have excluded cases with kidneys severely distorted with huge tumours, necrosis and shifted within the abdomen, as depicted in Fig. 3. For such diseased organs with prominent malignant tissue, the Dice coefficient indicated a lower match of around $30 \%$, but these represent only a tiny fraction of the overall cases studied. There is no agreed convention on whether to include tumourous tissue into the segmentation, and the decision is mostly task-dependent. In our case only healthy tissue had been labelled in the gold standard, yet the machine crossed the relatively weak boundaries between the tumour and the healthy tissue, and went on to label both, thus mis-segmenting the organ. Figure 4 illustrates this. 


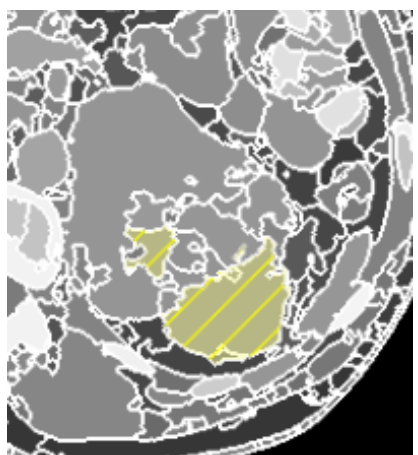

(a) Labelling approximation in the IPF includes part of the tumour.

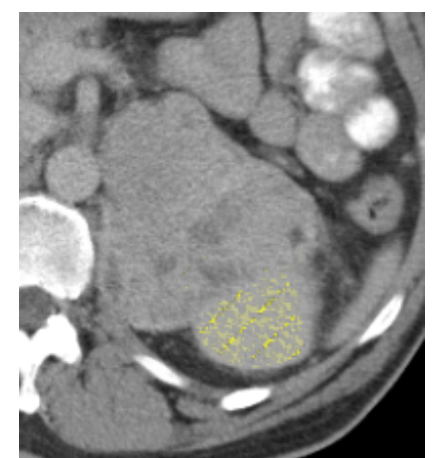

(b) Seeds for FMM have been extracted from the labelling approximation in (a).

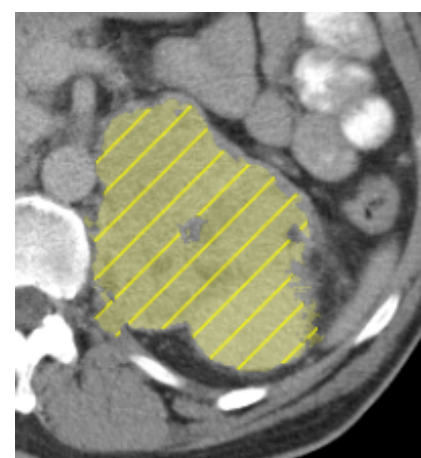

(c) FMM growth extended to include the malignant tissue in the kidney labelling.

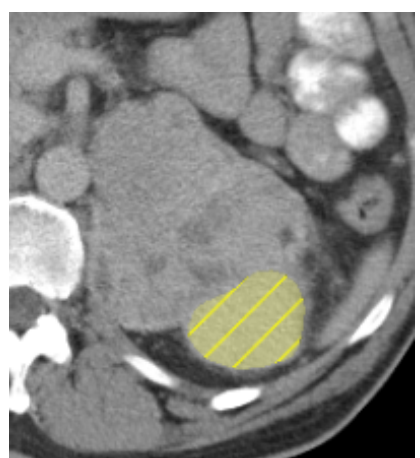

(d) Manually produced ground truth excludes voxels corresponding to the tumour.

Figure 4. Machine segmented kidney with big tumour vs. its manually labelled ground truth which excludes the tumorous tissue

The results of labelling the main abdominal organs in a 40 slice volume have been piped into multiple material Marching Cubes, ${ }^{33}$ automatically yielding the 3D reconstruction shown in Fig. 5. Once the 3D shapes are available, evaluation techniques (such as volume calculations, evaluation of thickness, or rate of growth) become straightforward to compute, as does preparation for 3D printing (see Fig. 5(c)).

On a laptop with average configuration our automated segmentation algorithm runs for 10-20 minutes for an image volume of 20 slices. Running it on a parallel super-computer would shorten the overall time, but we are keen to maintain our image analysis software tool to lowest configuration parameters so as to make it more readily accessible to clinicians.

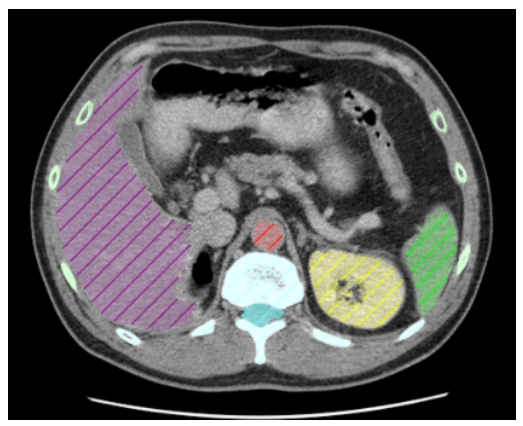

(a) Final results shown on a single axial CT slice

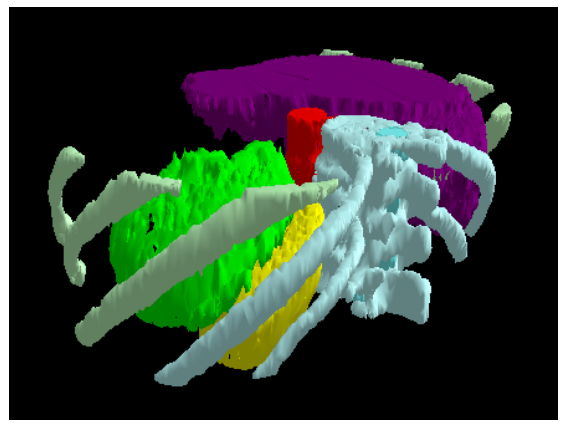

(b) 3D marching cubes reconstruction of labelled features

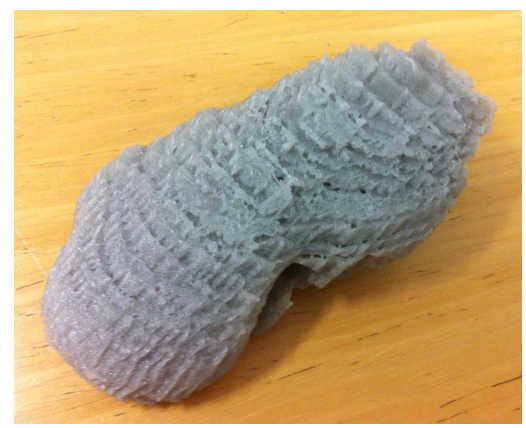

(c) 3D printed healthy kidney

Figure 5. 3D abdominal feature labelling

\section{CONCLUSIONS AND FUTURE WORK}

The novelty of seeding a front propagation method automatically from a carefully crafted hierarchical structure makes our segmentation entirely unsupervised and automated. Our current experiments on kidneys show a match between automated segmentation results and manually labelled gold standard of $90 \%$. This is similar to the state-of-the-art algorithms, ${ }^{31,32}$ albeit on different data. We are not aware of open access benchmarking data for the kidneys, nor for the abdomen.

Although our current quantitative results are reported for renal segmentation only, the method is easily extrapolated to other organs and tissue types, already shown in Fig. 5. Since new medical scanners produce data with increasingly higher resolution and accuracy, experiments on a wider class of volumes are needed, including on MRI data (which we have started tackling). 
All our results have been validated qualitatively by a human judge. Whilst the procedure is fully automated, in practice users (particularly clinicians) sometimes prefer to override some of the automatic features of the algorithms. For that consideration, all our software tools provide both automatic and manual parameter setting, providing a light level of supervision if desired. The overall time saver for the user is, in any case, the automation of the overall segmentation, leaving them the much less onerous option of fine tuning the final labelled volume as a post-processing task.

\section{ACKNOWLEDGMENTS}

We are grateful to Dr. Zoë Traill, of the Churchill Hospital Oxford, for providing us with abdominal CT scan data and for spending considerable time drawing gold standard contours with our software tool. Thanks to Samuel Littley for the 3D printed kidney model obtained automatically from our data.

\section{REFERENCES}

[1] Mimics ${ }^{\mathrm{TM}}$. biomedical materialise.com/mimics.

[2] Simpleware ${ }^{\mathrm{TM}}$. simpleware.com/software.

[3] Malladi, R., Sethian, J. A., and Vemuri, B. C., "Topology-independent shape modeling scheme," in [SPIE's 1993 International Symposium on Optics, Imaging, and Instrumentation], 246-258, International Society for Optics and Photonics (1993).

[4] Malladi, R., Sethian, J. A., and Vemuri, B. C., "Evolutionary fronts for topology-independent shape modeling and recovery," in [Computer Vision - ECCV'94], Eklundh, J.-O., ed., Lecture Notes in Computer Science 800, 1-13, Springer Berlin Heidelberg (1994).

[5] Malladi, R., Sethian, J. A., and Vemuri, B. C., "Shape modeling with front propagation: A level set approach," IEEE Transactions on Pattern Analysis and Machine Intelligence 17, 158-175 (Feb. 1995).

[6] Malladi, R., Sethian, J. A., and Vemuri, B. C., "A fast level set based algorithm for topology-independent shape modeling," Journal of Mathematical Imaging and Vision 6(2-3), 269-289 (1996).

[7] Malladi, R. and Sethian, J. A., "Level set methods for curvature flow, image enchancement, and shape recovery in medical images," in [Visualization and Mathematics], Hege, H.-C. and Polthier, K., eds., 329345, Springer Berlin Heidelberg (1997).

[8] Malladi, R. and Sethian, J. A., "An $\mathrm{O}(\mathrm{N} \log \mathrm{N})$ algorithm for shape modeling," Proceedings of the National Academy of Sciences 93(18), 9389-9392 (1996).

[9] Malladi, R. and Sethian, J. A., "Level set and fast marching methods in image processing and computer vision," in [Proceedings of International Conference on Image Processing], 1, 489-492, IEEE (Sept. 1996).

[10] Malladi, R. and Sethian, J. A., "A real-time algorithm for medical shape recovery," in [Sixth International Conference on Computer Vision], 304-310, IEEE (Jan. 1998).

[11] Campadelli, P., Casiraghi, E., and Pratissoli, S., "Fully automatic segmentation of abdominal organs from CT images using fast marching methods," in [21st IEEE International Symposium on Computer-Based Medical Systems, 2008. CBMS'08], 554-559, IEEE (June 2008).

[12] Campadelli, P., Casiraghi, E., and Pratissoli, S., "A segmentation framework for abdominal organs from CT scans," Artificial Intelligence in Medicine 50(1), 3-11 (2010).

[13] Sethian, J. A., [Level Set Methods and Fast Marching Methods: Evolving Interfaces in Computational Geometry, Fluid Mechanics, Computer Vision, and Materials Science], Cambridge Monographs on Applied and Computational Mathematics, Cambridge University Press (1999).

[14] Golodetz, S., Voiculescu, I., and Cameron, S., "A proposed decision-support system for (renal) cancer imaging," in [Proceedings of FBIT '07], 361-366 (Oct. 2007).

[15] Golodetz, S., Voiculescu, I., and Cameron, S., "Progress on a decision-support system for abdominal CT scans (work-in-progress paper)," in [Proceedings of HSI 2009], Bello, L. L. and Iannizzotto, G., eds., 116-119 (May 2009). Best Paper Award (Telemedicine and e-Health).

[16] Tidwell, A. S., "Advanced imaging concepts: A pictorial glossary of CT and MRI technology," Clinical Techniques in Small Animal Practice 14(2), 65-111 (1999). 
[17] Perona, P. and Malik, J., "Scale-space and edge detection using anisotropic diffusion," Pattern Analysis and Machine Intelligence, IEEE Transactions on 12(7), 629-639 (1990).

[18] Golodetz, S., Voiculescu, I., and Cameron, S., "Automatic spine identification in abdominal CT slices using image partition forests," in [Proceedings of 6th International Symposium on Image and Signal Processing and Analysis, 2009. ISPA 2009.], 117-122, IEEE (Sept. 2009).

[19] Golodetz, S. M., Nicholls, C., Voiculescu, I. D., and Cameron, S. A., "Two tree-based methods for the waterfall," Pattern Recognition (May 2014).

[20] Beucher, S., "Watershed, hierarchical segmentation and waterfall algorithm," in [Mathematical Morphology and Its Applications to Image Processing], Serra, J. and Soille, P., eds., Computational Imaging and Vision 2, 69-76, Springer Netherlands (1994).

[21] Sethian, J. A., "A fast marching level set method for monotonically advancing fronts," Proceedings of the National Academy of Sciences 93(4), 1591-1595 (1996).

[22] Sethian, J. A., "Fast marching methods," SIAM Review 41(2), 199-235 (1999).

[23] Yatziv, L., Bartesaghi, A., and Sapiro, G., "O(N) implementation of the fast marching algorithm," Journal of Computational Physics 212(2), 393-399 (2006).

[24] Rasch, C. and Satzger, T., "Remarks on the $\mathrm{O}(\mathrm{N})$ implementation of the fast marching method," IMA Journal of Numerical Analysis 29(3), 806-813 (2009).

[25] Yeghiazaryan, V. and Voiculescu, I., "The use of fast marching methods in medical image segmentation," Tech. Rep. CS-RR-15-07, Department of Computer Science, University of Oxford, Oxford, UK (2015).

[26] Gonzalez, R. C. and Woods, R. E., [Digital Image Processing], Prentice Hall (2008). 3rd edition.

[27] Dice, L. R., "Measures of the amount of ecologic association between species," Ecology 26(3), 297-302 (1945).

[28] Udupa, J. K., LeBlanc, V. R., Zhuge, Y., Imielinska, C., Schmidt, H., Currie, L. M., Hirsch, B. E., and Woodburn, J., "A framework for evaluating image segmentation algorithms," Computerized Medical Imaging and Graphics 30(2), 75-87 (2006).

[29] Cárdenes, R., de Luis-García, R., and Bach-Cuadra, M., "A multidimensional segmentation evaluation for medical image data," Computer Methods and Programs in Biomedicine 96(2), 108-124 (2009).

[30] Yeghiazaryan, V. and Voiculescu, I., "An overview of current evaluation methods used in medical image segmentation," Tech. Rep. CS-RR-15-08, Department of Computer Science, University of Oxford, Oxford, UK (2015).

[31] Cuingnet, R., Prevost, R., Lesage, D., Cohen, L. D., Mory, B., and Ardon, R., "Automatic detection and segmentation of kidneys in 3D CT images using random forests," in [Medical Image Computing and Computer-Assisted Intervention - MICCAI 2012], Ayache, N., Delingette, H., Golland, P., and Mori, K., eds., Lecture Notes in Computer Science 7512, 66-74, Springer Berlin Heidelberg (2012).

[32] Linguraru, M. G., Pura, J. A., Pamulapati, V., and Summers, R. M., "Statistical 4D graphs for multi-organ abdominal segmentation from multiphase CT," Medical Image Analysis 16(4), 904-914 (2012).

[33] Wu, Z. and Sullivan, J. M., "Multiple material marching cubes algorithm," International Journal for Numerical Methods in Engineering 58(2), 189-207 (2003). 